\title{
An annotated checklist of Egyptian mosses
}

\author{
Wagieh El-Saadawi', Hanaa M. Shabbara', Manal Ibrahim \\ Khalil $^{3}$ and Mai Ahmed Taha ${ }^{*}$ \\ ${ }^{1-4}$ Department of Botany, Faculty of Science, Ain Shams University, Cairo, \\ Egypt; e-mail: 'wagelsaadawi@yahoo.com, ${ }^{2}$ shabbarah@yahoo.com, \\ 3manalibrahim2000@yahoo.com, ${ }^{4}$ maitaha33@yahoo.com \\ *Corresponding author.
}

Wagieh El-Saadawi, Hanaa M. Shabbara, Manal Ibrahim Khalil and Mai Ahmed Taha, 2015. An annotated checklist of Egyptian mosses. Taeckholmia 35: 1-23.

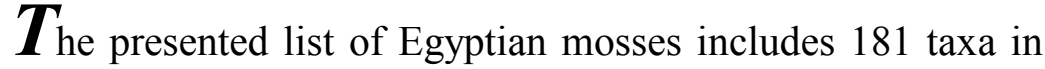
56 genera, 17 families and 10 orders. Synonyms reported only from Egypt are given in a separate list. The distribution of the 181 mosses in the 11, hitherto, surveyed phytogeographic territories of Egypt shows that S, Mm, Cai and $\mathrm{Di}$ are the richest territories regarding the number of recorded taxa. Pottiaceae, Bryaceae and Funariaceae dominate the flora. Pohlia lescuriana (Sull.) Ochi is a new record to Egypt. Other relevant annotations are also given.
\end{abstract}

Key words: Checklist, Egypt, Mosses, Pohlia lescuriana.

\section{Introduction}

The increasing interest in the taxonomy of the bryophytes, especially aimed at biodiversity conservation has stimulated the elaboration of updated and corrected checklists (Cortini 2001). During the last five decades six checklists of Egyptian mosses had been published by: Imam \& Ghabbour (1972); EL-Saadawi and Abou EL-Kheir (1973); El-Saadawi \& Badawi (1977); El-Saadawi et al. (1999); El-Saadawi et al. (2003) and Ros et al. (2013). Naturally the number of recorded mosses increased with time and the last list included 166 taxa.

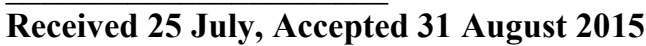


Recently five new papers (El-Saadawi et al. 2013 a, b; Ibrahim et al. 2013; El-Sakaty et al. 2014 and Ibrahim 2014) appeared, adding 14 records to the moss flora of the country. This paper, further adds Pohlia lescuriana (Sull.) Ochi as a new record to the country bringing the total number of mosses known from Egypt to 181 taxa (Table 1). These taxa belong to 56 genera (Table 1) in 17 families and 10 orders (Table 2); and collected from 11 out of the 15 phytogeographical territories of Egypt (Fig. 1).

To bring the new data on Egyptian mosses together in one piece of literature the present checklist was established appended by annotations and a list of synonyms. The presented data, as well, throw more light on the moss flora of each of the 11 surveyed phytogeographic territories of Egypt, the matter that was not covered in the latest published list (i.e. Ros et al. 2013).

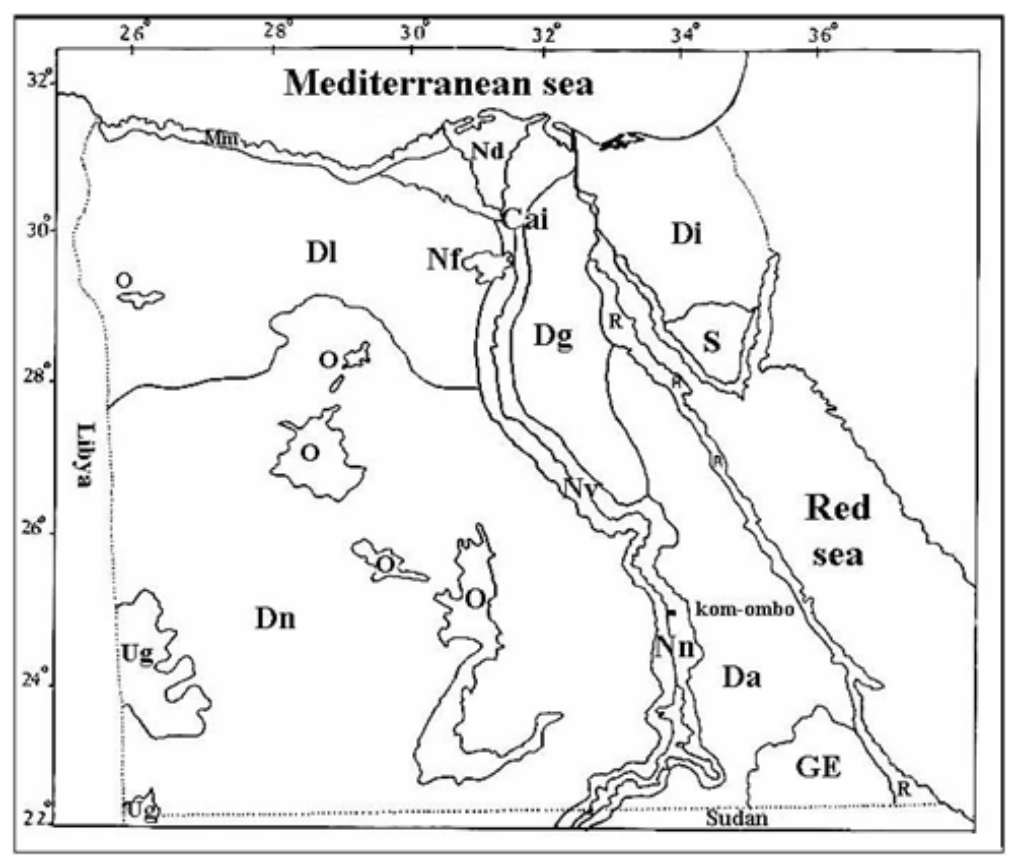

Fig. 1. A map showing phytogeographical territories of Egypt (after El-Saadawi et al. 2003; with little modification). Cai: Cairo area; Da: Arabian desert; Dg: Galala Desert; Di: Isthmic Desert; Dl: Libyan Desert; Dn: Nubian desert; GE: Gebel Elba; Mm: western Mediterranean coastal land (Mareotic sector); Nd: Nile Delta; Nf: Nile Fayoum; Nn: Nile Nubia, from Kom Ombo southwards to Egyptian boundaries with the Sudan including the areas now inundated by the waters of Lake Naser since 1965; Nv: Nile Valley, from Cairo-Giza to Kom Ombo; O: Oasis of the Nubian and Libyan Desert; R: Red Sea coastal plains; S: Southern Sinai massive (Sinai proper i.e. relatively high mountains, south of Isthmic desert); Ug: Gebel Uweinat. 
Table 1. Distribution of the 181 moss taxa belonging to 56 genera in the 11 surveyed phytogeographic territories of Egypt. $+=$ Present; * $=$ New record; ${ }^{\circ}=$ Reported without reference to any locality; $\mathbf{0}=$ Recorded only from greenhouses and gardens, $\bullet=$ Doubtful record. Abbreviations of names of territories are as given in Fig. 1.

\begin{tabular}{|c|c|c|c|c|c|c|c|c|c|c|c|}
\hline $\begin{array}{c}\text { Taxa } \\
\end{array}$ & Nn & Nv & Nd & $\mathbf{N f}$ & Cai & Dg & Di & $\mathbf{S}$ & GE & $\mathbf{O}$ & Mm \\
\hline \multicolumn{12}{|l|}{ 1. Aloina Kindb. (Pottiaceae) } \\
\hline A.aloides (Koch ex Schultz) Kindb. & & & & & & & + & + & & & + \\
\hline $\begin{array}{l}\text { A.ambigua (Bruch \&Schimp.) } \\
\text { Limpr. }\end{array}$ & & & & & & & + & + & & & + \\
\hline A.bifrons (De Not.) Delgad. & & & & & & & + & & & & + \\
\hline $\begin{array}{l}\text { A.brevirostris (Hook. \& Grev.) } \\
\text { Kindb. }\end{array}$ & & & & & & & & & & & + \\
\hline A.rigida (Hedw.) Limpr. & & & & & & & + & & & + & + \\
\hline \multicolumn{12}{|l|}{$\begin{array}{l}\text { 2. Anoectangium Schwägr. } \\
\text { (Pottiaceae) }\end{array}$} \\
\hline A.handelii Schiffn. & & & & & & & & + & & & \\
\hline \multicolumn{12}{|l|}{ 3. Archidium Brid. (Archidiaceae) } \\
\hline A.alternifolium (Hedw.) Mitt. & & & + & & & & & & & & + \\
\hline \multicolumn{12}{|l|}{ 4. Barbula Hedw. (Pottiaceae) } \\
\hline B.arcuata Griff & & & & + & & & & & & & \\
\hline B.bolleana (Müll.Hal.) Broth. & + & + & + & + & + & & & + & & & + \\
\hline B.convoluta Hedw. & + & & + & & & + & & + & & & \\
\hline B. convoluta var. sardoa Schimp. & & & + & & & & & & & & \\
\hline B.indica (Hook.) Spreng. & & + & + & & + & & & & & & \\
\hline B.unguiculata Hedw. & & + & + & + & + & + & & & & & + \\
\hline \multicolumn{12}{|l|}{$\begin{array}{l}\text { 5. Brachymenium Schwägr. } \\
\text { (Bryaceae) }\end{array}$} \\
\hline $\begin{array}{l}\text { B. exile (Dozy \&Molk.) Bosch. \& } \\
\text { Sande Lac. }\end{array}$ & & & & & & & + & + & & + & + \\
\hline \multicolumn{12}{|l|}{$\begin{array}{l}\text { 6.Brachytheciastrum Ignatov \& } \\
\text { Huttunen (Brachytheciaceae) }\end{array}$} \\
\hline \multicolumn{12}{|l|}{$\begin{array}{l}\text { B.velutinum (Hedw.) Ignatov \& } \\
\text { Huttunen }\end{array}$} \\
\hline \multicolumn{12}{|l|}{$\begin{array}{l}\text { B. velutinum var. salicinum } \\
\text { (Schimp.) Ochyra \& Żarnowiec }\end{array}$} \\
\hline \multicolumn{12}{|l|}{$\begin{array}{l}\text { 7. Brachythecium Schimp. } \\
\text { (Brachytheciaceae) }\end{array}$} \\
\hline B.rivulare Schimp. & & & + & & + & & & & & & \\
\hline \multicolumn{12}{|l|}{ B.umbilicatumJur. \&Milde } \\
\hline \multicolumn{12}{|l|}{ 8. Bryum Hedw. (Bryaceae) } \\
\hline B.apiculatum Schwägr. & & & + & & & & & & & + & \\
\hline B.argenteum Hedw. & & & & & + & + & + & + & & + & + \\
\hline B. cellulare Hook. & & & & & + & + & & - & + & & \\
\hline B.dichotomum Hedw. & & + & + & + & + & + & + & + & & + & + \\
\hline B.elegans Nees & & & & & & & & + & & + & \\
\hline B. funkii Schwägr. & & & & & & + & + & + & & + & \\
\hline B.gemmiparum De Not. & & & & + & + & & & + & & + & + \\
\hline B.kunzei Hornsch. & & & & & + & & & - & & - & + \\
\hline B.radiculosum Brid. & & & + & & + & & + & + & & + & + \\
\hline B.ruderale Crundw. \&Nyholm & & & & & & & & + & & 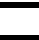 & \\
\hline 므 B.sauteri Bruch \&Schimp. & & & & & + & & & & & & \\
\hline B.schleicheri DC. & & & & & & & & + & & & \\
\hline B.subapiculatum Hampe & & + & & & + & & & + & & + & \\
\hline
\end{tabular}




\begin{tabular}{|c|c|c|c|c|c|c|c|c|c|c|c|}
\hline Taxa & Nn & $\mathbf{N v}$ & Nd & Nf & Cai & Dg & Di & $\mathbf{S}$ & GE & $\mathbf{O}$ & $\mathbf{M m}$ \\
\hline B.turbinatum (Hedw.) Turner & & & & & & & & + & & + & + \\
\hline 9-Ceratodon Brid. (Ditrichaceae) & & & & & & & & & & & \\
\hline C. purpureus (Hedw.) Brid. & & & & + & & & & & & & \\
\hline $\begin{array}{l}\text { 10. Cinclidotus P.Beauv. } \\
\text { (Pottiaceae) }\end{array}$ & & & & & & & & & & & \\
\hline C.riparius (Host ex Brid.) Arn. & & & & & & & & + & & & \\
\hline 11.Crossidium Jur. (Pottiaceae) & & & & & & & & & & & \\
\hline C. aberrans Holz. \&E.B.Bartram & & & & & & & + & + & & & + \\
\hline C. crassinervium (De Not.) Jur. & & & & & & & + & & & + & + \\
\hline C. geheebii (Broth.) Broth. & & & & & & & + & + & & & \\
\hline C. laevipilum Thér. \&Trab. & & & & & & & + & & & & + \\
\hline $\begin{array}{l}\text { C. laxefilamentosum } \\
\text { W.Frey\&Kürschner }\end{array}$ & & & & & & & + & & & & + \\
\hline C. squamiferum (Viv.) Jur. & & & & & & & + & + & & + & \\
\hline $\begin{array}{l}\text { C. squamiferum var. pottioideum } \\
\text { (De Not.) Mönk. }\end{array}$ & & & & & & & & + & & & \\
\hline $\begin{array}{l}\text { 12.Dialytrichia (Schimp.) Limpr. } \\
\text { (Pottiaceae) }\end{array}$ & & & & & & & & & & & \\
\hline D. mucronata (Brid.) Broth. & & & & & & & & + & & & \\
\hline $\begin{array}{l}\text { 13.Dichodontium Schimp. } \\
\text { (Rhabdoweisiaceae) }\end{array}$ & & & & & & & & & & & \\
\hline D. pellucidum (Hedw.) Schimp. & & & & & + & & & & & + & \\
\hline $\begin{array}{l}\text { 14.Dicranella (Müll.Hal.) Schimp. } \\
\text { (Dicranaceae) }\end{array}$ & & & & & & & & & & & \\
\hline D. rufescens (Dicks.) Schimp. & & & & + & & & & & & & \\
\hline 15.Didymodon Hedw. (Pottiaceae) & & & & & & & & & & & \\
\hline D. acutus (Brid.) K.Saito & & & & & & & + & + & & & + \\
\hline $\begin{array}{l}\text { D. australasiae (Hook. \& Grev.) } \\
\text { R.H.Zander }\end{array}$ & & & & & & + & + & + & & & \\
\hline D. cordatus Jur. & & & & & & & & & & & + \\
\hline D. fallax (Hedw.) R.H.Zander & & & + & + & & & + & + & & + & + \\
\hline $\begin{array}{l}\text { D. ferrugineus (Schimp. ex Besch.) } \\
\text { M.O.Hill }\end{array}$ & & & & & & & & & & & + \\
\hline D. insulanus (De Not.) M.O.Hill & & & + & & & + & & & & & + \\
\hline D. luridus Hornsch. & & & + & + & + & + & & + & & & + \\
\hline D. rigidulus Hedw. & & & & & & & + & + & & + & + \\
\hline D. sinuosus (Mitt.) Delogne & & & & + & & & & & & & + \\
\hline D. spadiceus (Mitt.) Limpr. & & & & & & + & & + & & & \\
\hline D. tectorum (Müll.Hal.)K.Saito. & & & & & & & & & & & + \\
\hline D. tophaceus (Brid.) Lisa & + & + & + & + & + & + & & + & + & + & + \\
\hline $\begin{array}{l}\text { D. umbrosus (Müll.Hal.) } \\
\text { R.H.Zander }\end{array}$ & & & & & & & & + & & & \\
\hline D. vinealis (Brid.) R.H.Zander & & & + & & & & + & + & & + & + \\
\hline $\begin{array}{l}\text { 16. Drepanocladus (Müll.Hal.) } \\
\text { G.Roth (Amblystegiaceae) }\end{array}$ & & & & & & & & & & & \\
\hline D. aduncus (Hedw.) Warnst. & & & & & & & & + & & & \\
\hline $\begin{array}{l}\text { 17.Encalypta Hedw. } \\
\text { (Encalyptaceae) }\end{array}$ & & & & & & & & & & & \\
\hline E.intermedia Jur. & & & & & & & & + & & & \\
\hline E.vulgaris Hedw. & & & & & & & & + & & & \\
\hline $\begin{array}{l}\text { 18. Entosthodon Schwägr. } \\
\text { (Fuariaceae) }\end{array}$ & & & & & & & & & & & \\
\hline E.attenuatus (Dicks.) Bryhn & & & & & & & + & + & & & \\
\hline
\end{tabular}


An annotated checklist of Egyptian mosses

\begin{tabular}{|c|c|c|c|c|c|c|c|c|c|c|c|}
\hline Taxa & Nn & Nv & Nd & $\mathbf{N f}$ & Cai & Dg & Di & $\mathbf{S}$ & GE & $\mathbf{O}$ & Mm \\
\hline E.cf. commutatus Durieu\& Mont. & & & & & & & & & & & + \\
\hline E.convexus (Spruce) Brugués & & & & & & & & + & & & \\
\hline E.curvi-apiculatus Müll.Hal. & & + & & & & & & & & & \\
\hline E.durieui Mont. & & & & & + & & + & + & + & & \\
\hline E.fascicularis (Hedw.) Müll.Hal. & & & & & + & & + & + & & & \\
\hline E.handelii (Schiffn.) Laz. & & & & & + & + & & & & & \\
\hline E.muhlenbergii (Turner) Fife & & & & & + & & & + & + & & \\
\hline E.niloticus Schimp. & & & & & + & + & & & & & \\
\hline E.obtusus (Hedw.) Lindb. & & & & & + & & & & & & \\
\hline $\begin{array}{l}\text { E.planoconvexus (E.B. Bartram) } \\
\text { Grout }\end{array}$ & & & & & & & + & & & & \\
\hline E.pulchellus (H.Philib.) Brugués & & & & & & & + & + & + & & \\
\hline $\begin{array}{l}\text { 19.Eucladium Bruch \&Schimp. } \\
\text { (Pottiaceae) }\end{array}$ & & & & & & & & & & & \\
\hline $\begin{array}{l}\text { E.verticillatum (With.) Bruch \& } \\
\text { Schimp. }\end{array}$ & & & & & + & & & + & & & \\
\hline $\begin{array}{l}\text { 20.Fissidens Hedw. } \\
\text { (Fissidentaceae) }\end{array}$ & & & & & & & & & & & \\
\hline F.arnoldii R.Ruthe & & & & & & & + & + & + & & \\
\hline F.bryoides Hedw. & & & + & & + & + & + & & & & + \\
\hline $\begin{array}{l}\text { F.crassipes Wilson ex Bruch } \\
\text { \&Schimp. }\end{array}$ & & & & & + & & + & & & & \\
\hline $\begin{array}{l}\text { F. crassipes subsp. warnstorfii } \\
\text { (M.Fleisch.) Brugg.-Nann. }\end{array}$ & & + & + & & + & & & & & & + \\
\hline F.crispus Mont. & & + & & & + & & & + & & & + \\
\hline${ }^{\circ}$ F.fontanus (Bach.Pyl.) Steud. & & & & & & & & & & & \\
\hline F.gymnandrus Büse & & & & + & & & & & & & \\
\hline F.viridulus (Sw.) Wahlenb. & + & + & + & + & + & + & & & + & & + \\
\hline$F$. viridulus var. viridulus & & & & & + & & & & & & \\
\hline $\begin{array}{l}\text { 21.Fontinalis Hedw. } \\
\text { (Fontinalaceae) }\end{array}$ & & & & & & & & & & & \\
\hline F.hypnoides C.Hartm. & + & & & & & & & & & & \\
\hline 22.Funaria Hedw. (Funariaceae & & & & & & & & & & & \\
\hline$F$. anomala Jur. & & & & & + & & & & & & \\
\hline F. hygrometrica Hedw. & + & + & + & + & + & + & + & + & & + & + \\
\hline F.limbata (Müll. Hal.) Broth. & & & + & & & & & & & & \\
\hline F.microstoma Bruch ex Schimp. & & & & & & & & & & + & \\
\hline F.nubica Müll.Hal. & + & & & & & & & & & & \\
\hline F.sickenbergeri Müll.Hal. & & & & & & & & & & + & \\
\hline 23. Grimmia Hedw. (Grimmiace & & & & & & & & & & & \\
\hline G.anodon Bruch \& Schimp. & & & & & & & & + & & & \\
\hline G.crinita Brid. & & & & & & & & + & & & \\
\hline G.laevigata (Brid.) Brid. & & & & & & & & + & & & \\
\hline G.orbicularis Bruch ex Wilson & & & & & & & & + & & & \\
\hline $\begin{array}{l}\text { 24. Gymnostomum Nees \& } \\
\text { Hornsch. (Pottiaceae) }\end{array}$ & & & & & & & & & & & \\
\hline G.aeruginosum $\mathrm{Sm}$. & & & + & + & + & & & + & & + & \\
\hline G.calcareum Nees\&Hornsch. & & + & + & + & & + & & + & + & & + \\
\hline G.mosis (Lorentz) Jur. \&Milde & & & & & & & + & + & & & \\
\hline G.vridulum Brid. & & + & & & + & + & + & + & & & + \\
\hline $\begin{array}{l}\text { 25. Gyroweisia Schimp. } \\
\text { (Pottiaceae) }\end{array}$ & & & & & & & & & & & \\
\hline G.reflexa (Brid.) Schimp. & & & + & & + & + & & + & & & \\
\hline
\end{tabular}


Wagieh El-Saadawi et al.

\begin{tabular}{|c|c|c|c|c|c|c|c|c|c|c|c|}
\hline Taxa & Nn & Nv & Nd & Nf & Cai & Dg & $\overline{\mathrm{Di}}$ & $\mathbf{S}$ & GE & $\mathbf{O}$ & Mm \\
\hline G.tenuis (Hedw.) Schimp. & & & + & & & & & + & & & \\
\hline 26 Hennediella Paris (Pottiaceae) & & & & & & & & & & & \\
\hline H.stanfordensis (Steere) Blockeel & & & & & & & & & & & + \\
\hline $\begin{array}{l}\text { 27.Hygroamblystegium Loeske } \\
\text { (Amblystegiaceae) }\end{array}$ & & & & & & & & & & & \\
\hline هH. varium (Hedw.) Mönk. & & & & & + & & & & & & + \\
\hline $\begin{array}{l}\text { 28.Hymenostylium Brid. } \\
\text { (Pottiaceae) }\end{array}$ & & & & & & & & & & & \\
\hline H.crassinervium Broth. \& Dixon & & & & & & & & + & & & \\
\hline H.recurvirostrum (Hedw.) Dixon & & & + & & & & & + & & & \\
\hline $\begin{array}{l}\text { 29.Imbribryum N.Pedersen } \\
\text { (Bryaceae) }\end{array}$ & & & & & & & & & & & \\
\hline $\begin{array}{l}\text { I.alpinum (Huds.ex With.) } \\
\text { N.Pedersen }\end{array}$ & + & & & + & + & & & + & & & \\
\hline I.mildeanum (Jur.) J.R. Spence & & & & & & & & + & & & \\
\hline $\begin{array}{l}\text { 30.Leptobarbula Schimp. } \\
\text { (Pottiaceae) }\end{array}$ & & & & & & & & & & & \\
\hline L.berica (De Not.) Schimp. & & & & & & + & & & & & \\
\hline $\begin{array}{l}\text { 31.Leptobryum (Bruch \&Schimp.) } \\
\text { Wilson (Meesiaceae) }\end{array}$ & & & & & & & & & & & \\
\hline L. pyriforme (Hedw.) Wilson & & & + & & + & & & & & & + \\
\hline $\begin{array}{l}\text { 32.Leptodictyum (Schimp.) } \\
\text { Warnst. (Amblystegiaceae) }\end{array}$ & & & & & & & & & & & \\
\hline L.riparium (Hedw.) Warnst. & & & & & + & & & + & & & \\
\hline $\begin{array}{l}\text { 33.Microbryum Schimp. } \\
\text { (Pottiaceae) }\end{array}$ & & & & & & & & & & & \\
\hline M.davallianum (Sm.) R.H.Zander & & & & & & & + & & & & + \\
\hline $\begin{array}{l}\text { M.starckeanum (Hedw.) } \\
\text { R.H.Zander }\end{array}$ & & & & & + & & + & + & & & + \\
\hline $\begin{array}{l}\text { 34.Microeurhynchium Ignatov \& } \\
\text { Vanderpoorten } \\
\text { (Brachytheciaceae) }\end{array}$ & & & & & & & & & & & \\
\hline $\begin{array}{l}\text { M. pumilum (Wilson) Ignatov } \\
\text { \&Vanderpoorten }\end{array}$ & & + & & & + & & & & & & \\
\hline $\begin{array}{l}\text { 35. Orthotrichum Hedw. } \\
\text { (Orthotrichaceae) }\end{array}$ & & & & & & & & & & & \\
\hline O.rupestre Schleich. Ex Schwägr. & & & & & & & & + & & & \\
\hline $\begin{array}{l}\text { 36.Philonotis Brid. } \\
\text { (Bartramiaceae) }\end{array}$ & & & & & & & & & & & \\
\hline P.fontana (Hedw.) Brid. & & & & & + & & & & & & \\
\hline P.hastata (Duby) Wijk \& Margad. & & & + & + & + & + & & & & + & \\
\hline P.marchica (Hedw.) Brid. & & & & + & + & & & & & & \\
\hline $\begin{array}{l}\text { 37.Physcomitrium (Brid.) Brid. } \\
\text { (Funariaceae) }\end{array}$ & & & & & & & & & & & \\
\hline P.eurystomum Sendtn. & & & & & + & + & & & & & \\
\hline $\begin{array}{l}\text { P. eurystomum subsp. acuminatum } \\
\text { (Bruch \&Schimp.) Giacom. }\end{array}$ & & & & & + & + & & & & & \\
\hline P.immersum Sull. & & & & & & & + & & & & \\
\hline P.niloticum (Delile) Müll. Hal. & & + & + & + & + & + & & & + & & \\
\hline $\begin{array}{l}\text { P.pyriforme (Hedw.) Bruch } \\
\text { \&Schimp. }\end{array}$ & + & & + & & + & + & & & & & \\
\hline 38.Plaubelia Brid. (Pottiaceae) & & & & & & & & & & & \\
\hline P.sprengelii (Schẅagr) R.H. Zander & & & & & & & + & & & & \\
\hline $\begin{array}{l}\text { 39.Pleuridium Rabenh. } \\
\text { (Ditrichaceae) }\end{array}$ & & & & & & & & & & & \\
\hline
\end{tabular}


An annotated checklist of Egyptian mosses

\begin{tabular}{|c|c|c|c|c|c|c|c|c|c|c|c|}
\hline $\begin{array}{c}\text { Taxa } \\
\end{array}$ & Nn & $\mathbf{N v}$ & Nd & $\mathbf{N f}$ & Cai & Dg & Di & $\mathbf{S}$ & GE & $\mathbf{O}$ & Mm \\
\hline 口 P.subulatum (Hedw.) Rabenh. & & & & & + & & & & & & + \\
\hline 40.Pohlia Hedw. (Mniaceae) & & & & & & & & & & & \\
\hline $\begin{array}{l}\text { P. atropurpurea (Wahlenb.) } \\
\text { H.Lindb. }\end{array}$ & & & & & & & & + & & & \\
\hline $\begin{array}{l}\text { P.korbiana (Müll.Hal.) } \\
\text { Wijk\&Margad. }\end{array}$ & & & & & & & & & & + & \\
\hline *P.lescuriana (Sull.) Ochi & & & & + & & & & & & & \\
\hline P.melanodon (Brid.) A.J.Shaw & & & & & & & & + & & & + \\
\hline P.nutans (Hedw.) Lindb. & & & & & + & & & & & & + \\
\hline $\begin{array}{l}\text { P.wahlenbergii } \\
\text { (F.Weber\&D.Mohr) A.L.Andrews }\end{array}$ & & & & & & & & + & & & \\
\hline $\begin{array}{l}\text { 41.Pseudocrossidium R.S.Williams } \\
\text { (Pottiaceae) }\end{array}$ & & & & & & & & & & & \\
\hline $\begin{array}{l}\text { P.hornschuchianum (Schultz) } \\
\text { R.H.Zander }\end{array}$ & & + & & & & & + & + & & & + \\
\hline $\begin{array}{l}\text { 42.Pterygoneurum Jur. } \\
\text { (Pottiaceae) }\end{array}$ & & & & & & & & & & & \\
\hline P. subsessile (Brid.) Jur. & & & & & & & + & & & & \\
\hline $\begin{array}{l}\text { 43.Ptychostomum Hornsch. } \\
\text { (Bryaceae) }\end{array}$ & & & & & & & & & & & \\
\hline $\begin{array}{l}\text { P.archangelicum (Bruch } \\
\text { \&Schimp.) J.R.Spence }\end{array}$ & & & & & & & & + & & + & \\
\hline $\begin{array}{l}\text { P.bornholmense (Wink. \& R. } \\
\text { Ruthe) Holyoak \& N. Pedersen }\end{array}$ & & & & & & & & + & & & \\
\hline $\begin{array}{l}\text { P.capillare (Hedw.) } \\
\text { D.T.Holyoak\&N.Pedersen }\end{array}$ & & & & & + & + & + & + & & + & \\
\hline $\begin{array}{l}\text { P.creberrimum (Taylor) J.R. } \\
\text { Spence \& H.P. Ramsay }\end{array}$ & & & & & & & & + & & & \\
\hline $\begin{array}{l}\text { P.imbricatulum (Müll.Hal.) } \\
\text { D.T.Holyoak\&N.Pedersen }\end{array}$ & & & + & & + & + & + & + & & + & + \\
\hline P. pallens (Sw.) J.R.Spence & & & & & & & & + & & & \\
\hline $\begin{array}{l}\text { P.pseudotriquetrum (Hedw.) } \\
\text { J.R.Spence\&H.P.Ramsay }\end{array}$ & & & & & & & & + & & + & \\
\hline $\begin{array}{l}\text { P. pseudotriquetrum var. bimum } \\
\text { (Schreb.) D.T.Holyoak\&N.Pedersen }\end{array}$ & & & & & & & & + & & & \\
\hline $\begin{array}{l}\text { P.rubens (Mitt.) } \\
\text { Holyoak\&N.Pedersen }\end{array}$ & & & & & & & & + & & & \\
\hline $\begin{array}{l}\text { P. torquescens (Bruch \&Schimp.) } \\
\text { Ros \& Mazimpaka }\end{array}$ & & & & & & + & & + & & + & \\
\hline $\begin{array}{l}\text { 44.Racomitrium Brid. } \\
\text { (Grimmiaceae) }\end{array}$ & & & & & & & & & & & \\
\hline R.aciculare (Hedw.) Brid. & & & & & & & & + & & & \\
\hline $\begin{array}{l}\text { 45.Rhynchostegiella (Schimp.) } \\
\text { Limpr. (Brachytheciaceae) }\end{array}$ & & & & & & & & & & & \\
\hline R.tenella (Dicks.) Limpr. & & & & & & & & + & & & \\
\hline $\begin{array}{l}\text { 46. Rhynchostegium Schimp. } \\
\text { (Brachytheciaceae) }\end{array}$ & & & & & & & & & & & \\
\hline R.riparioides (Hedw.) Cardot & & & & & & & & + & & & \\
\hline $\begin{array}{l}\text { 47.Schistidium Bruch \& Schimp. } \\
\text { (Grimmiaceae) }\end{array}$ & & & & & & & & & & & \\
\hline S.agassizii Sull. \&Lesq. & & & & & & & & + & & & \\
\hline $\begin{array}{l}\text { S.apocarpum (Hedw.) Bruch } \\
\text { \&Schimp. }\end{array}$ & & & & & & & & + & & & \\
\hline $\begin{array}{l}\text { 48.Sciuro-hypnum Hampe } \\
\text { (Brachytheciaceae) }\end{array}$ & & & & & & & & & & & \\
\hline
\end{tabular}


Wagieh El-Saadawi et al.

\begin{tabular}{|c|c|c|c|c|c|c|c|c|c|c|c|}
\hline Taxa & Nn & Nv & Nd & Nf & Cai & $\overline{D g}$ & Di & $\mathbf{S}$ & GE & $\mathbf{O}$ & Mm \\
\hline \multicolumn{12}{|l|}{$\begin{array}{l}\text { ○S.plumosum (Hedw.) } \\
\text { Ignatov\&Huttunen }\end{array}$} \\
\hline \multicolumn{12}{|l|}{$\begin{array}{l}\text { 49.SplachnobryumMüll.Hal. } \\
\text { (Pottiaceae) }\end{array}$} \\
\hline $\begin{array}{l}\text { S. limbatum D.H. Norris \& R.H. } \\
\text { Zander }\end{array}$ & & & & & & & & & & & + \\
\hline S.obtusum (Brid.) Müll.Hal. & & & + & & & & & & & & \\
\hline \multicolumn{12}{|l|}{ 50.Syntrichia Brid. (Pottiaceae) } \\
\hline S.fragilis (Taylor) Ochyra & & & + & & & & & + & & & \\
\hline \multicolumn{12}{|l|}{$\begin{array}{l}\text { S.handelii (Schiffn.) } \\
\text { S.Agnew\&Vondr. }\end{array}$} \\
\hline S.rigescens (Broth. \&Geh.) Ochyra & & & & & & & & + & & & \\
\hline S.virescens (De Not.) Ochyra & & & & & & & & + & & & \\
\hline \multicolumn{12}{|l|}{$\begin{array}{l}\text { 51.Timmiella (De Not.) Limpr. } \\
\text { (Pottiaceae) }\end{array}$} \\
\hline T. barbuloides (Brid.) Mönk. & & & & & & & + & + & + & & \\
\hline \multicolumn{12}{|l|}{$\begin{array}{l}\text { 52.Tortella (Müll.Hal.) Limpr. } \\
\text { (Pottiaceae) }\end{array}$} \\
\hline T. flavovirens (Bruch.) Broth. & & & & & & & & & & & + \\
\hline T.humilis (Hedw.) Jenn. & & & & & & & + & & & & \\
\hline T.inclinata (R.Hedw.) Limpr. & & & & & & & & & & + & \\
\hline T.inflexa (Bruch) Broth. & & & & & & & & & & & + \\
\hline T.nitida (Lindb.) Broth. & & + & & & & & & & + & & + \\
\hline T.tortuosa (Hedw.) Limpr. & & & & & & + & & & & & \\
\hline \multicolumn{12}{|l|}{ 53.Tortula Hedw. (Pottiaceae) } \\
\hline T.atrovirens $(\mathrm{Sm}$.$) Lindb.$ & & & & & & & + & + & & & + \\
\hline T.brevissima Schiffn. & & & & & & & & + & & & + \\
\hline T.canescens Mont. & & & & & & & & + & & & \\
\hline T.caucasica Broth. & & & & & & & & & & & + \\
\hline T.cuneifolia (Dicks.) Turner & & & & & & & & & & & + \\
\hline T.inermis (Brid.) Mont. & & & & & & & + & + & & & \\
\hline T.kneuckeri Broth. \&Geh. & & & & & & & & + & & & \\
\hline $\begin{array}{l}\text { T.marginata (Bruch \&Schimp.) } \\
\text { Spruce }\end{array}$ & & & & & & & & & & & + \\
\hline T.muralis Hedw. & & & + & & + & + & + & & & & + \\
\hline T.muralis var.aestiva (Hedw.) Brid. & & & & & & & & & & & + \\
\hline T.pallida (Lindb.) R.H.Zander & & & & & & & & & & & + \\
\hline T.plinthobia (Sull. \&Lesq.) Broth. & & & & & & & & & & + & + \\
\hline T.vahliana (Schultz) Mont. & & & & & & & & & & & + \\
\hline T.wilsonii (Hook.) R.H.Zander & & & & & & & & & & & + \\
\hline \multicolumn{12}{|l|}{$\begin{array}{l}\text { 54.Trichostomum Bruch } \\
\text { (Pottiaceae) }\end{array}$} \\
\hline T.brachydontium Bruch & & & + & & & & + & & & & \\
\hline T.crispulum Bruch & & & + & & & & + & + & & & + \\
\hline \multicolumn{12}{|l|}{ 55.Weissia Hedw. (Pottiaceae) } \\
\hline W.controversa Hedw. & & & & & & & & + & & & \\
\hline \multicolumn{12}{|l|}{$\begin{array}{l}\text { W.controversa } \text { var. crispata } \\
\text { (Nees\&Hornsch.) Nyholm }\end{array}$} \\
\hline W.longifolia Mitt. & & & & & & & & & & & + \\
\hline \multicolumn{12}{|l|}{ W.sinaloensis E.B.Bartram } \\
\hline $\begin{array}{l}\text { 56.Zygodon Hook. \& Taylor } \\
\text { (Orthotrichaceae) }\end{array}$ & & & & & & & & & & & \\
\hline
\end{tabular}




\begin{tabular}{|l|c|c|c|c|c|c|c|c|c|c|c|}
\hline \multicolumn{1}{|c|}{ Taxa } & Nn & Nv & Nd & Nf & Cai & Dg & Di & S & GE & O & Mm \\
\hline Z.obtusifolius Hook. & & & & + & & & & & & & \\
\hline Total & $\mathbf{9}$ & $\mathbf{1 7}$ & $\mathbf{3 6}$ & $\mathbf{2 2}$ & $\mathbf{5 1}$ & $\mathbf{3 0}$ & $\mathbf{4 6}$ & $\mathbf{9 5}$ & $\mathbf{1 1}$ & $\mathbf{3 2}$ & $\mathbf{6 5}$ \\
\hline
\end{tabular}

\section{Annotations}

A. Based on Table 1 above, the following annotations were listed.

- The phytogeographic territories of Egypt can be arranged, regarding their relative richness in moss speices in descending order as follows: $\mathrm{S}, \mathrm{Mm}$, Cai, Di, Nd, O, Dg, Nf, Nv, GE and Nn.

- Fontinalis hypnoides (Fontinalaceae) was recorded only once from River Nile during its course in the Nile Nubia.

- The most widespread species in the moss flora of Egypt are Didymodon tophaceus and Funaria hygrometrica (10 territories each), Bryum dichotomum (9 territories), Fissidens viridulus (8 territories), Barbula bolleana, Gymnostomum calcareum and Ptychostomum imbricatulum (7 territories each), Barbula unguiculata, Bryum argenteum, Bryum radiculosum, Didymodon fallax, Didymodon luridus, Gymnostomum viridulum and Physcomitrium niloticum (6 territories each), Bryum gemmiparum, Didymodon vinealis, Fissidens bryoides, Gymnostomum aeruginosum, Philonotis hastata, Ptychostomum capillare and Tortula muralis (5 territories each).

- Bryum sauteri, Hygroamblystegium varium and Pleuridium subulatum are the only mosses recorded here from only green houses and gardens.

- Fissidens fontanus and Sciuro-hypnum plumosum (=Brachythecium plumosum) were not referred to any particular locality in the original publications (Brotherus 1924; Bilewsky 1965); cited only as Egypt and Sinai respectively, therefore given the symbol ${ }^{\circ}$ in the present list.

- According to Ros et al. (2013) Bilewsky (1965) reported Brachythecium plumosum (Hedw.) Schimp. from Sinai (Northern Sinai, i.e. Di) without locality and included Brachythecium umbilicatum Jur. \& Milde as a synonym of this species. At present both species are accepted taxonomically, but only Brachythecium umbilicatum has been reported from Egypt, by Geheeb (1904). Therefore, the presence of Sciurohypnum plumosum (= B. plumosum) in Egypt is doubtful, therefore given the symbol $\downarrow$ in the present list.

- During the preparation of the present list, it was noticed by a mere chance (while surveying earlier works on Egyptian mosses) that 
illustrations given for Bryum elegans from Nf by Badawi et al. (1987) are quite different from those in other published reference works, therefore we re-examined carefully the specimen of $\mathrm{Nf}$ and found that it belongs in fact to Pohlia lescuriana which represents a new record to Egypt as reported in the present list.

- In the list published by El-Saadawi et al. (2003) Splachnobryum obtusum was mentioned as occurring in Nf, however, this is incorrect, therefore this species is excluded from Nf territory in the present list.

- The report of Bryum subapiculatum in the present list as occurring in Cairo is recorded for the first time in this territory after Farag (2015).

- Eighty-six out of the 181 taxa were recorded in only one territory each. While only 21 taxa were recorded in five or more territories each. This may be attributed to the great variation of both physical and chemical properties of microhabitats in different territories of Egypt (Taha 2010) and probably also to the rarity of sporophyte production (Abd El-Khaleq 2004) by Egyptian mosses.

B. Based on Table 2 below, the following annotations were listed.

- The bryologically distinguished territories are S (the only territory for Encalyptales and Grimmiales) and Nf (the only territory for Dicranaceae).

- Three families (Pottiaceae, Bryaceae and Funariaceae) are represented in the 11 terrirtories while Fissidentaceae is represented in 10 territories. The remaining thirteen families are relatively poorly represented (1-5 territories each).

- The largest family is Pottiaceae followed by Bryaceae.

- The 10 orders can be arranged in a descending order regarding the number of taxa representing each as follows: Pottiales- 84, Bryales- 37, Funariales- 23, Hypnales- 12, Fissidentales- 9, Grimmiales- 7, Dicranales- 4, Encalyptales- 2, Orthotrichales- 2 and Archidiales- 1. 


\section{An annotated checklist of Egyptian mosses}

Table 2: Names of the 10 orders and 17 families representing the moss flora of Egypt and the number of taxa under each, and their distribution in the 11 surveyed phytogeographic territories of the country. Abbreviations of names of territories are as given in Fig. 1.

\begin{tabular}{|c|c|c|c|c|c|c|c|c|c|c|c|c|}
\hline \multirow{2}{*}{ Orders and families } & \multicolumn{11}{|c|}{ Territories/No. of taxa } & \multirow{2}{*}{$\begin{array}{l}\text { Total } \\
\text { No. of } \\
\text { taxa }\end{array}$} \\
\hline & Nn & $\mathbf{N v}$ & Nd & Nf & Cai & Dg & Di & $\mathbf{S}$ & GE & $\mathbf{O}$ & Mm & \\
\hline 1. Pottiales & 3 & 8 & 21 & 9 & 11 & 13 & 29 & 43 & 4 & 11 & 45 & 84 \\
\hline Family: Pottiaceae & 3 & 8 & 21 & 9 & 11 & 13 & 29 & 43 & 4 & 11 & 45 & 84 \\
\hline 2. Bryales & 1 & 2 & 6 & 6 & 16 & 8 & 7 & 26 & 1 & 17 & 11 & 37 \\
\hline Family 1: Bryaceae & 1 & 2 & 4 & 4 & 11 & 7 & 7 & 23 & 1 & 15 & 8 & 28 \\
\hline Family 2: Bartramiaceae & & & 1 & 2 & 3 & 1 & & & & 1 & & 3 \\
\hline Family 3: Meesiaceae & & & 1 & & 1 & & & & & & 1 & 1 \\
\hline Family 4: Mniaceae & & & & & 1 & & & 3 & & 1 & 2 & 5 \\
\hline 3. Funariales & 3 & 3 & 4 & 2 & 12 & 7 & 7 & 7 & 4 & 3 & 2 & 23 \\
\hline Family: Funariaceae & 3 & 3 & 4 & 2 & 12 & 7 & 7 & 7 & 4 & 3 & 2 & 23 \\
\hline 4. Hypnales & & 2 & 1 & & 4 & & & 7 & & & 1 & 12 \\
\hline Family 1: Brachytheciaceae & & 1 & 1 & & 2 & & & 5 & & & & 8 \\
\hline Family 2: Amblystegiaceae & & & & & 2 & & & 2 & & & 1 & 3 \\
\hline Family 3: Fontinalaceae & & 1 & & & & & & & & & & 1 \\
\hline 5. Fissidentales & 1 & 3 & 3 & 2 & 6 & 2 & 3 & 2 & 2 & & 4 & 9 \\
\hline Family: Fissidentaceae & 1 & 3 & 3 & 2 & 6 & 2 & 3 & 2 & 2 & & 4 & 9 \\
\hline 6. Grimmiales & & & & & & & & 7 & & & & 7 \\
\hline Family: Grimmiaceae & & & & & & & & 7 & & & & 7 \\
\hline 7. Dicranales & & & & 2 & 2 & & & & & 1 & 1 & 4 \\
\hline Family 1: Dicranaceae & & & & 1 & & & & & & & & 1 \\
\hline Family 2: Ditrichaceae & & & & 1 & 1 & & & & & & 1 & 2 \\
\hline Family 3: Rhabdoweisiaceae & & & & & 1 & & & & & 1 & & 1 \\
\hline 8. Encalyptales & & & & & & & & 2 & & & & 2 \\
\hline Family: Encalyptaceae & & & & & & & & 2 & & & & 2 \\
\hline 9. Orthotrichales & & & & 1 & & & & 1 & & & & 2 \\
\hline Family: Orthotrichaceae & & & & 1 & & & & 1 & & & & 2 \\
\hline 10. Archidiales & & & 1 & & & & & & & & 1 & 1 \\
\hline Family: Archidiaceae & & & 1 & & & & & & & & 1 & 1 \\
\hline
\end{tabular}


List of synonyms of moss taxa reported only from Egypt, based on ElSaadawi et al. (1999) and Ros et al. (2013) arranged alphabetically Aloina aloides (Schultz) Kindb.var. ambigua (Bruch \& Schimp. \& W. Gümbel) E.J. Craig. = Aloina ambigua (Bruch \& Schimp.) Limpr.

Aloina pilifera (De Not.) H.A. Crum \& Steere = Aloina bifrons (De Not.) Delgad.

Aloina rigida (Hedw.) Limpr.var. ambigua (Bruch \& Schimp. \& W. Gümbel) E.J. Craig. = Aloina ambigua (Bruch \& Schimp.) Limpr.

Aloina rigida (Hedw.) Limpr. var. pilifera (De Not.) Limpr. = Aloina bifrons (De Not.) Delgad.

Amblystegium burnatii J.J. Amann. = Microeurhynchium pumilum (Wilson) Ignatov\&Vanderpoorten

Amblystegium riparium (Hedw.) Bruch, Schimp. \& W. Gümbel = Leptodictyum riparium (Hedw.) Warnst.

Amblystegium varium (Hedw.) Lindb. = Hygroamblystegium varium (Hedw.) Mönk.

Anisothecium rufescens (With.) Lindb. = Dicranella rufescens (Dicks.) Schimp.

Astomum crispum (Hedw.) Hampe $=$ Weissia longifolia Mitt.

Barbula aaronis (Lorentz) Hilp. = Didymodon australasiae (Hook. \& Grev.) R.H. Zander

Barbula acuta (Brid.) Brid. = Didymodon acutus (Brid.) K. Saito.

Barbula alexandrina Lorentz $=$ Tortella nitida (Lindb.) Broth.

Barbula aloides (Koch ex Schultz) Bruch =Aloina aloides (Koch ex Schultz) Kindb.

Barbula bescherellei Sauerb. = Didymodon rigidulus Hedw.

Barbula cylindrica (Taylor) Schimp. = Didymodon insulanus (De Not.) M.O. Hill

Barbula ehrenbergii (Lorentz) M.Fleisch. = Barbula bolleana (Müll. Hal.) Broth.

Barbula gracilis (Schleich.) Schwägr. var. viridis Bruch \& Schimp. = Didymodon acutus (Brid.) K. Saito.

Barbula hornschuchiana Schultz = Pseudocrossidium hornschuchianum (Schultz) R.H. Zander

Barbula inermis [Lorentz] $=$ Tortula inermis (Brid.) Mont.

Barbula membranifolia Hook. = Crossidium squamiferum (Viv.) Jur.

Barbula muralis var. alexandrina Müll. Hal.= Tortella nitida (Lindb.) Broth. 
Barbula muralis var. incana Bruch, Schimp. \& W. Gümbel = Tortula muralis Hedw.

Barbula nitida Lindb. = Tortella nitida (Lindb.) Broth.

Barbula reflexa (Brid.) Brid.= Didymodon ferrugineus (Schimp. ex Besch.) M.O. Hill

Barbula sardoa (Schimp.) J.P. Frahm = Barbula convoluta var. sardoa Schimp.

Barbula sinuosa (Mitt.) Grav. = Didymodon sinuosus (Mitt.) Delogne.

Barbula tectorum Müll. Hal. = Didymodon tectorum (Müll. Hal.) K. Saito.

Barbula tophacea (Brid.) Mitt. = Didymodon tophaceus (Brid.) Lisa

Barbula unguiculata fo. cuspidata (Schultz) Mönk. = Barbula unguiculata Hedw.

Barbula unguiculata fo. obtusifolia Mönk. = Barbula unguiculata Hedw.

Barbula unguiculata fo. robusta (Lindb.) Podp. = Barbula unguiculata Hedw.

Barbula unguiculata var. cuspidata (Schultz) Brid. = Barbula unguiculata Hedw.

Barbula unguiculata var. robusta Lindb. = Barbula unguiculata Hedw.

Barbula vinealis Brid. = Didymodon vinealis (Brid.) R.H. Zander

Barbula vinealis (Brid.) subsp. cylindrica (Taylor) Podp. $=$ Didymodon insulanus (De Not.) M.O. Hill

Barbula vinealis var. cylindrica (Taylor) Boulay nom. illeg. = Didymodon insulanus (De Not.) M.O. Hill

Barbula vinealis var. flaccida Bruch \& Schimp. = Didymodon insulanus (De Not.) M.O. Hill

Brachythecium velutinum (Hedw.) (Bruch \& Schimp. \& W. Gümbel) = Brachytheciastrum velutinum (Hedw.) Ignatov\& Huttunen

Brachythecium velutinum (Hedw.) (Bruch \& Schimp. \& W. Gümbel) var. venustum (De Not.) Arcang. = Brachytheciastrum velutinum var. salicinum (Schimp.) Ochyra \& Zarnowiec

Brachythecium venustum (De Not.)De Not. = Brachytheciastrum velutinum var. salicinum (Schimp.) Ochyra \& Zarnowiec

Bryum alpinum Huds. ex With subsp. gemmiparum (De Not.) Kindb. = Bryum gemmiparum De Not.

Bryum alpinum Huds. ex With. = Imbribryum alpinum (Huds.ex With.) N.Pedersen

Bryum alpinum Huds. ex With. var. viride Husn. = Imbribryum alpinum (Huds.ex With.) N.Pedersen 
Bryum alpinum subsp. gemmiparum f. sinaica Podp.= Bryum gemmiparum De Not.

Bryum alpinum With var. gemmiparum (De Not.) Lindb. = Bryum gemmiparum De Not.

Bryum argenteum Hedw. var. lanatum (P. Beauv.) Hampe = Bryum argenteum Hedw.

Bryum aschersonii Müll. Hal. = Ptychostomum capillare (Hedw.) D.T.Holyoak \& N.Pedersen

Bryum atropurpureum Wahlenb.= Pohlia atropurpurea (Wahlenb.) H. Lindb.

Bryum atrovirens Brid. = Bryum subapiculatum Hampe

Bryum badium (Brid.) Schimp. = Ptychostomum imbricatulum (Müll.Hal.)

D.T.Holyoak \& N.Pedersen

Bryum bicolor Dicks.,1801= Bryum dichotomum Hedw.

Bryum caespiticium Hedw. = Ptychostomum imbricatulum (Müll.Hal.)

D.T.Holyoak \& N.Pedersen

Bryum caespiticium Hedw. subsp. comense (Schimp.) J.J. Amann = Ptychostomum imbricatulum (Müll.Hal.) D.T.Holyoak \& N.Pedersen

Bryum caespiticium Hedw. var. badium Brid. = Ptychostomum imbricatulum (Müll.Hal.) D.T.Holyoak \& N.Pedersen

Bryum caespiticium Hedw. var. comense (Schimp.) Husn. = Ptychostomum imbricatulum (Müll.Hal.) D.T.Holyoak \& N.Pedersen

Bryum caespiticium Hedw. var. imbricatum Bruch, Schimp. \& W. Gümbel = Bryum kunzei Hornsch.

Bryum capillare (Hedw.) subsp. elegans (Nees) Lindb. = Bryum elegans Nees

Bryum capillare Hedw. = Ptychostomum capillare $($ Hedw.) D.T.Holyoak \& N.Pedersen

Bryum capillare Hedw. subsp. torquescens (De Not.) Kindb. = Ptychostomum torquescens (Bruch \&Schimp.) Ros\&Mazimpaka

Bryum comense Schimp. = Ptychostomum imbricatulum (Müll.Hal.) D.T.Holyoak \& N.Pedersen

Bryum creberrimum Taylor $=$ Ptychostomum creberrimum (Taylor) J.R. Spence \& H.P. Ramsay

Bryum gemmiparum De Not. var. sinaica Geh.= Bryum gemmiparum De Not.

Bryum inclinatum (Brid.) Bland = Ptychostomum archangelicum (Bruch \&Schimp.) J.R.Spence 
Bryum korbianum Müll. Hal. = Pohlia korbiana (Müll. Hal.) Wijk \& Margad.

Bryum murorum (Schimp.) Berk. = Bryum radiculosum Brid.

Bryum pseudotriquetrum (Hedw.) P. Gaertn., G. Mey. \& Scherb. = Ptychostomum pseudotriquetrum (Hedw.) J.R.Spence \& H.P.Ramsay

Bryum pseudotriquetrum (Hedw.) P. Gaertn., G. Mey. \& Scherb. var. bimum (Brid.) Lilj. = Ptychostomum pseudotriquetrum var. bimum (Schreb.) D.T.Holyoak \& N.Pedersen

Bryum sacrum (Lorentz) Müll. Hal. = Bryum turbinatum (Hedw.) Turner Bryum syriacum Lorentz = Bryum turbinatum $($ Hedw.) Turner

Bryum syriacum var. humile Schiffn. = Bryum turbinatum (Hedw.) Turner Bryum torquescense Bruch ex De Not. = Ptychostomum torquescens (Bruch \&Schimp.) Ros\&Mazimpaka

Bryum turbinatum Hedw. = Bryum turbinatum (Hedw.) Turner

Bryum turbinatum $\mathrm{Sw} .=$ Bryum turbinatum (Hedw.) Turner

Cinclidotus mucronatus (Brid.) Machado-Guim. = Dialytrichia mucronata (Brid.) Broth.

Crossidium chloronotos auct. non (Brid.) Limpr. =Crossidium crassinervium (De Not.) Jur.

Desmatodon convolutus (Brid.) Grout $=$ Tortula atrovirens $(\mathrm{Sm}$.) Lindb.

Desmatodon plinthobius Sull. \& Lesq. = Tortula plinthobia (Sull. \& Lesq.) Broth.

Dichodontium pellucidum (Hedw.) Schimp. fo. fagimontanum C.E.O. Jensen = Dichodontium pellucidum (Hedw.) Schimp.

Dichodontium pellucidum (Hedw.) Schimp. var. fagimontanum (Brid.) Schimp. = Dichodontium pellucidum (Hedw.) Schimp.

Didymodon aaronis (Lorentz) J. Guerra = Didymodon australasiae (Hook. \& Grev.) R.H. Zander

Didymodon acutus (Brid.) K. Saito f. viridis (Bruch, Schimp. \& W. Gümbel) Podp. =Didymodon acutus (Brid.) K. Saito.

Didymodon ceratodentus (ceratodonteus) (Müll. Hal.) Dixon $=$ Didymodon tophaceus (Brid.) Lisa

Didymodon ehrenbergii (Lorentz) Kindb. = Barbula bolleana (Müll. Hal.) Broth.

Didymodon fallax var reflexus (Brid.) R.H.Zander = Didymodon ferrugineus (Schimp. ex Besch.)

Didymodon haussknechtii (Jur. \& Milde) Broth. = Didymodon australasiae (Hook. \& Grev.) R.H. Zander 
Didymodon rigidulus Hedw. var. gracilis (Schleich. ex Hook. \& Grev.) R.H. Zander = Didymodon acutus (Brid.) K. Saito.

Didymodon trifarius (Hedw.) Röhl. = Didymodon luridus Hornsch.

Didymodon trivialis (Müll. Hal.) J. Guerra = Didymodon umbrosus (Müll. Hal.) R.H. Zander

Encalypta vulgaris Hedw. var. mediolanensis Warnst. = Encalypta intermedia Jur.

Entosthodon niloticus (Delile) Schimp. = Entosthodon niloticus Schimp.

Entosthodon pallescens Jur.= Entosthodon duriaei Mont.

Entosthodon pallescens Jur. fo. foliis solidioribus = Entosthodon duriaei Mont.

Entosthodon templetonii (Sm.) Schwägr. = Entosthodon attenuatus (Dicks.) Bryhn

Eucladium verticillatum forma [Lorentz $]=$ Eucladium verticillatum (With.) Bruch \& Schimp.

Eucladium verticillatum L. = Eucladium verticillatum (With.) Bruch \& Schimp.

Eurhynchium pumilum (Wilson) Schimp. = Microeurhynchium pumilum (Wilson) Ignatov\&Vanderpoorten

Fissidens alexandrinus Lorentz $=$ Fissidens crassipes subsp. warnstorfii (M.Fleisch.) Brugg.-Nann.

Fissidens bambergeri Schimp. ex Milde = Fissidens viridulus (Sw. ex anon.) Wahlenb. var.viridulus

Fissidens bambergeri var. aegyptiacus Renauld \& Cardot $=$ Fissidens crassipes subsp. warnstorfii (M.Fleisch.) Brugg.-Nann.

Fissidens bryoides Hedw. var. gymnandrus (Büse) R. Ruthe $=$ Fissidens gymnandrus Büse

Fissidens bryoides Hedw. subsp. viridulus (Sw.) Kindb. = Fissidens viridulus (Sw.) Wahlenb.

Fissidens crassipes Wilson ex Bruch, Schimp. \& W. Gümbel var. philibertii Besch.= Fissidens crassipes subsp. warnstorfii (M.Fleisch.) Brugg.-Nann.

Fissidens cyprius Jur. $=$ Fissidens crispus Mont.

Fissidens impar Mitt. = Fissidens viridulus (Sw.) Wahlenb.

Fissidens julianus (Sav.) Schimp. = Fissidens fontanus (Bach. Pyl.) Steud.

Fissidens limbatus Sull. $=$ Fissidens crispus Mont.

Fissidens mnevidis J.J. Amann $=$ Fissidens crassipes subsp. warnstorfii (M.Fleisch.) Brugg.-Nann. 
Fissidens obtusifolius Wilson $=$ Fissidens arnoldii R. Ruthe Fissidens viridulus subsp. bambergeri (Schimp.) Kindb. $=$ Fissidens viridulus (Sw.) Wahlenb. var. viridulus

Fissidens viridulus (Sw.) Wahlenb. var. bambergeri (Schimp.) Waldh. = Fissidens viridulus (Sw.) Wahlenb. var. viridulus

Funaria attenuata (Dicks.) Lindb. = Entosthodon attenuatus (Dicks.) Bryhn

Funaria calcarea Wahlenb. var. mediterranea (Lindb.) C.E.O. Jensen \& Medelius = Entosthodon muhlenbergii (Turner) Fife

Funaria convexa Spruce $=$ Entosthodon convexus (Spruce) Brugués

Funaria curvi-apiculata (Müll.Hal.) Broth. $=$ Entosthodon curviapiculatus Müll. Hal.

Funaria dentata Corme var. mediterranea (Lindb.) Limpr. = Entosthodon muhlenbergii (Turner) Fife

Funaria fascicularis (Hedw.) Lindb. = Entosthodon fascicularis (Hedw.) Müll. Hal.

Funaria handelii Schiffn.= Entosthodon handelii (Schiffn.) Laz.

Funaria hygrometrica Hedw. var. calvescens (Schwägr.) Mont. = Funaria hygrometrica Hedw.

Funaria hygrometrica Hedw. var. intermedia Warnst. = Funaria hygrometrica Hedw.

Funaria hygrometrica Hedw. var. minor (Delile) Brid. = Funaria hygrometrica Hedw.

Funaria hygrometrica Hedw. var. muralis Huebener $=$ Funaria hygrometrica Hedw.

Funaria hygrometrica Hedw. var. patula Bruch, Schimp. $=$ Funaria hygrometrica Hedw.

Funaria mediterranea Lindb. = Entosthodon muhlenbergii (Turner) Fife Funaria minor Delile = Funaria hygrometrica Hedw.

Funaria muhlenbergii Turner $=$ Entosthodon muhlenbergii (Turner) Fife Funaria nilotica Broth. = Entosthodon niloticus Schimp.

Funaria nilotica (Schimp. ex Müll. Hal.) Broth.= Entosthodon niloticus Schimp.

Funaria obtusa (Hedw.) Lindb. = Entosthodon obtusus (Hedw.) Lindb.

Funaria pallescens ( Jur.) Lindb. = Entosthodon duriaei Mont.

Funaria pulchella $\mathrm{H}$. Philib. = Entosthodon pulchellus (H. Philib.) Brugués

Grimmia alpicola $\mathrm{Sw} .=$ Schistidium agassizii Sull. \& Lesq. 
Grimmia anodon var. sinaitica Renauld \& Cardot = Grimmia anodon Bruch \& Schimp.

Grimmia apocarpa L. = Schistidium apocarpum (Hedw.) Bruch \& Schimp. Grimmia campestris Burch ex Hook. = Grimmia laevigata (Brid.) Brid.

Grimmia leucophaea Grev. = Grimmia laevigata (Brid.) Brid.

Grimmia sinaica Bruch \& Schimp. = Grimmia orbicularis Bruch ex Wilson Grimmia sinaica Hampe. = Grimmia crinita Brid.

Gymnostomum calcareum Nees \& Hornsch. var. muticum Boulay = Gymnostomum calcareum Nees \& Hornsch.

Gymnostomum calcareum Nees \& Hornsch. var. viridulum (Brid.) Bruch \& Schimp. $=$ Gymnostomum viridulum Brid.

Gymnostomum niloticum Delile = Physcomitrium niloticum (Delile) Müll. Hal.

Gymnostomum recurvirostre Hedw. $=$ Hymenostylium recurvirostrum (Hedw.) Dixon

Gyroweisia aaronis (Lorentz) Par. = Didymodon australasiae (Hook. \& Grev.) R.H. Zander

Gyroweisia mosis (Lorentz) Paris = Gymnostomum mosis (Lorentz) Jur. \& Milde

Gyroweisia rohlfsiana (Müll. Hal) Paris = Gymnostomum viridulum Brid. Hydrogonium ehrenbergii (Lorentz) A. Jaeger = Barbula bolleana (Müll. Hal.) Broth.

Hymenostylium rupestre Schwägr. = Gymnostomum aeruginosum Sm.

Hymenostylium verticillatum [Hart] = Eucladium verticillatum (With.) Bruch \& Schimp.

Hyophila stanfordensis (Steere) Smith \& Whitehouse $=$ Hennediella stanfordensis (Steere) Blockeel

Hypnum aduncum L. = Drepanocladus aduncus (Hedw.) Warnst.

Hypnum ruscifolium Neck. = Rhynchostegium riparioides (Hedw.) Cardot

Hypnum rusciforme Neck. $=$ Rhynchostegium riparioides (Hedw.) Cardot

Hypnum tenellum Dicks. = Rhynchostegiella tenella (Dicks.) Limpr.

Hypnum velutinum L. = Brachytheciastrum velutinum (Hedw.) Ignatov\& Huttunen

Microbryum starckeanum (Hedw.) R.H. Zander var. brachyodus (Bruch, Schimp. \& W .Gümbel) R.H. Zander= Microbryum starckeanum (Hedw.) R.H. Zander

Micropoma niloticum (Delile) Lindb. = Physcomitrium niloticum (Delile) Müll. Hal. 
Microstegium niloticum (Delile) Lindb. = Physcomitrium niloticum (Delile) Müll. Hal.

Mniobryum carneum ( L.) Limpr. = Pohlia melanodon (Brid.) A.J. Shaw.

Mniobryum delicatulum (Hedw.) Dixon = Pohlia melanodon (Brid.) A.J. Shaw.

Philonotis evanidinervis M. Fleisch. = Philonotis hastata (Duby) Wijk \& Marg

Philonotis glabriuscula kindb. pp. $=$ Philonotis fontana (Hedw.) Brid.

Philonotis glabriuscula kindb. pp.=Philonotis marchica (Hedw.) Brid.

Philonotula glabriuscula Müll. Hal. = pp. Philonotis fontana (Hedw.) Brid.

Philonotula glabriuscula Müll. Hal. = pp. Philonotis marchica (Hedw.)

Brid.

Philonotula marchica (Hedw.) Brid. fo. rivularis (Warnst.) Mönk. = Philonotis marchica (Hedw.) Brid.

Philonotis obtusata Müll. Hal. = Philonotis hastata (Duby) Wijk \& Margad.

Physcomitrium acuminatum (Schleich.) Bruch, Schimp. \& W. Gümbel = Physcomitrium eurystomum Sendtn.

Physcomitrium eurystomum Sendtn. fo. acuminatum (Bruch, Schimp. \& W.

Gümbel) Löske $=$ Physcomitrium eurystomum Sendtn.

Physcomitrium niloticum (Delile) Müll. Hal. = Entosthodon niloticus Schimp.

Physcomitrium niloticum Lindb. = Physcomitrium niloticum (Delile) Müll. Hal.

Physcomitrium sesostris Lorentz $=$ Physcomitrium niloticum (Delile) Müll. Hal.

Platyhypnidium rusciforme (Neck.) Fleisch. = Rhynchostegium riparioides (Hedw.) Cardot

Pohlia carnea (Schimp.) Lindb. = Pohlia melanodon (Brid.) A.J. Shaw.

Pottia intermedia (Turner) Fürnr.= Tortula caucasica Broth.

Pottia mutica Venturi= Microbryum starckeanum (Hedw.) R.H. Zander

Pottia pallida Lindb. = Tortula pallida (Lindb.) R.H.Zander

Pottia pallida Lindb. var. longicuspis Warnst. = Tortula pallida (Lindb.)

R.H.Zander

Pottia starckeana (Hedw.) Müll. Hal. = Microbryum starckeanum (Hedw.)

R.H. Zander

Pottia starckeana agg. (Hedw.) Müll Hal. = Microbryum starckeanum (Hedw.) R.H. Zander 
Pottia venusta Jur. = Tortula pallida (Lindb.) R.H.Zander

Pottia wilsonii (Hook.) Bruch, Schimp. \& W. Gümbel = Tortula wilsonii (Hook.) R.H. Zander

Rhynchostegiella algiriana (Brid.) Broth. = Rhynchostegiella tenella (Dicks.) Limpr.

Rhynchostegium rusciforme [Lorentz] = Rhynchostegium riparioides (Hedw.) Cardot

Rhynchostegium tenellum [Lorentz] = Rhynchostegiella tenella (Dicks.) Limpr.

Schistidium alpicola (Hedw.) Limpr. = Schistidium agassizii Sull. \& Lesq. Semibarbula orientalis (F. Weber) Wijk \& Margad. = Barbula indica (Hook.) Spreng.

Streblotrichum convolutum (Hedw.) P.Beauv. = Barbula convoluta Hedw. Syntrichia inermis (Brid.) Bruch = Tortula inermis (Brid.) Mont.

Systegium crispum (Hedw.) Hampe = Weissia longifolia Mitt.

Timmiella barbula (Schwägr.) Limpr. = Timmiella barbuloides (Brid.) Mönk.

Tortula fragilis (Taylor) Ochyra = Syntrichia fragilis (Taylor) Ochyra

Tortula modica R.H. Zander = Tortula caucasica Broth.

Tortula muralis Hedw. fo. incana (Bruch, Schimp. \& W. Gümbel) Sapjegin $=$ Tortula muralis Hedw.

Tortula muralis Hedw. fo. obcordata (Schimp.) Mönk. = Tortula muralis Hedw.

Tortula muralis Hedw. var. obcordata (Schimp.) Limpr. = Tortula muralis Hedw.

Tortula muralis Hedw. var. incana Bruch \& Schimp. = Tortula muralis Hedw.

Tortula pallida (Lindb.) R.H. Zander var. longicuspis (Warnst.) R.H. Zander $=$ Tortula pallida (Lindb.) R.H.Zander

Tortula plinthobia (Sull. \& Lesq.) Austin = Tortula plinthobia (Sull. \& Lesq.) Broth.

Tortula rigescens Broth. \& Geh.= Syntrichia rigescens (Broth. \&Geh.) Ochyra

Trichostomopsis aaronis (Lorentz) S. Agnew \& Tonwnsend = Didymodon australasiae (Hook. \& Grev.) R.H. Zander

Trichostomum aaronis Lorentz = Didymodon australasiae (Hook. \& Grev.)

R.H. Zander

Trichostomum aciculare P. Beauv. = Racomitrium aciculare (Hedw.) Brid. 
Trichostomum cylindricum (Brid.) C.Müll. = Oxystegus tenuirostris (Hook. \& Taylor) Lindb.

Trichostomum ehrenbergii Lorentz = Barbula bolleana (Müll. Hal.) Broth. Trichostomum mosis Lorentz $=$ Gymnostomum mosis (Lorentz) Jur. \& Milde

Trichostomum tophaceum fo. foliis magis acuminatis = Didymodon tophaceus (Brid.) Lisa

Webera carnea $[$ Lorentz] $=$ Pohlia melanodon (Brid.) A.J. Shaw.

Webera sacra Lorentz = Bryum turbinatum (Hedw.) Turner

Webera sinaitica Lorentz = Bryum turbinatum (Hedw.) Turner

Weissia rohlfsiana Müll. Hal. = Gymnostomum viridulum Brid. 


\section{References}

Abd EL-Khaleq, M.W. 2004: Life history of some Mosses from Cairo region. M.Sc. Thesis, Ain Shams University. 205 p.

Badawi, A., W. EL-Saadawi and Shabbara, H. 1987: On the mosses of EL-Fayum Region, Egypt. Ain Shams Sci. Bull. 26 B: 357 372.

Bilewsk, F. 1965: Moss flora of Israel. Nova Hedwigia 9: 335-434.

Brotherus, V.F. 1924: In: Engler A. \& Prantl K., Die Natürlichen Pflanzenfamilien 10. Berlin, Duncher and Humboldt.

Cortini, P.C. 2001: Checklist of the Mosses of Italy- Fl. Medit. 11: 21107.

EL-Saadawi, W. and Abou EL-Kheir, W. 1973: On some Egyptian mosses and the algal flora in their habitats. Proc. Egypt Acad. Sci. 26: $125-136$.

EL-Saadawi, W., Abou-Salama, U. and Taha, M. 2013 a: Mosses of the Egyptian conservation areas: III. Two new Pottiaceae records to Saint Catherine Protected Area and Egypt. Täeckholmia 33: 37-46.

EL-Saadawi, W. and Badawi. A. 1977: The moss flora of Egypt. I. order Pottiales. Publ. Cairo Univ. Herb. 7 \& 8:181-206.

EL-Saadawi, W., Badawi. A. , Shabbara, H., Abou-Salama, U.Y. and Refai, M.S. 1999: An updated list of Egyptian mosses. Täeckholmia; 19 (2): 77-96.

EL-Saadawi, W., Shabbara, H., Abou-Salama, U.Y. and Refai, M.S. 2003: Mosses of different phytogeographical territories of Egypt. Bocconea 16 (1): 133-146.

EL-Saadawi, W. Shabbara, H. and El-Sakaty, S.I. 2013 b: Mosses of the Egyptian conservation areas: II. Omayed Protected Area. Cryptogamie, Bryologie 34 (1): 1-11.

El-Sakaty, S.I., Magdy, M., El-Atroush, Mohamed, M. and AbouSalama, U. 2014: DNA Barcoding of Three Bryum Hedw. Taxa. Egyptian Journal of Botany. Special issue for the fourth international conference of Botany and Microbiological Science: 53-63. 
Farag, M. 2015: Morphological Plasticity Of Some Moss Species In Response To Various Environmental Conditions Via In Vitro Culture. M.Sc. Thesis, Ain Shams University. 148p.

Geheeb, A. 1904: Bryophyta. In: Kneucker A., Botanische Ausbeute einer Reise durch die Sinaihalbinsel vom 27. Marz bis 13. April 1902. Allge. Botan. Zeitung 1904: 4-5.

Ibrahim, M. 2010: Studies on family Pottiaceae (Musci) Menoufiya Province, Nile Delta, Egypt. Ph. D. Thesis, Ain Shams University. 205 p.

Ibrahim, M. 2014: Funaria limbata (Müll. Hal.) Broth. (Funariaceae); A new record to Egyptian mosses. Egypt. Jour. Bot. Special issue for the fourth international conference of Botany and Microbiological Science:1-9.

Ibrahim, H., Kamel, W., Abou-Salama, U. and Gamal-Eldin, E. 2013: The moss flora of Saint Katherine Protected Area, South Sinai, with eight new records. Täeckholmia 33: 19-36.

Imam, M. \& Ghabour, S. 1972. A contribution to the Moss Flora of Egypt. Bot. Notiser 125 (4): 518-522.

Ros, R.M., Mazimpaka, V. Abou-Salama, U., Aleffi, M., Blockeel, T.L., Brugués,M., Cros, R.M. , Dia, M.G., Dirkse, G.M., Draper,I., El-Saadawi,W., Erdă̆,A., Ganeva,A., Gabriel,R., González-Mancebo,J.M., Granger,C.,Herrnstadt, I.,Hugonnot, V., Khalil, K., Kürschner, H., Losada-Lima,A., Luis,L., Mifsud,S., Privitera, M., Puglisi, M., Sabovljević,M., Sérgio,C., Shabbara, H., Sim-Sim,M., Sotiaux,A., Tacchi, R. , Vanderpoorten, A. and Werner, O. 2013: Mosses of the Mediterranean, an annotated checklist. Cryptogamie, Bryologie 34 (2): 99-283.

Smith, A.J.E. 2004: The moss flora of Britain and Ireland. Cambridge University, Press.

Taha, M. 2010: Studies on family Pottiaceae (Musci) in El-Sharkyia Province. M.Sc. Thesis, Botany Department, Faculty of Science, Ain Shams University. 261 pp.

2014: Studies on the bryoflora of some wadis in Saint Catherine protected area. Ph.D.Thesis, Botany Department, Faculty of Science, Ain Shams University. 357 p. 